Draft: February 22, 2019

\title{
Broken Isotropy from a Linear Modulation of the Primordial Perturbations
}

\author{
Christopher Gordon ${ }^{1}$ \\ cgordon@kicp.uchicago.edu
}

\begin{abstract}
A linear modulation of the primordial perturbations is proposed as an explanation for the observed asymmetry between the northern and southern hemispheres of the Wilkinson Microwave Anisotropy Probe (WMAP) data. A cut sky, reduced resolution third year "Internal Linear Combination" (ILC) map was used to estimate the modulation parameters. A foreground template and a modulated plus unmodulated monopole and dipole were projected out of the likelihood. The effective chi squared was reduced by nine for three extra parameters. The mean galactic colatitude and longitude, of the modulation, with $68 \%, 95 \%$ and $99.7 \%$ confidence intervals were $56_{-17-35-51}^{+17+36+65}$ and $63_{-26-58-213}^{+28+59+105}$. The mean percentage change of the variance, across the pole's of the modulation, was $62_{-18-35-47}^{+18+35+57}$. Implications of these results and possible generating mechanisms are discussed.
\end{abstract}

Subject headings: cosmic microwave background, early universe, large-scale structure of universe

\section{Introduction}

A fundamental assumption of cosmology is that the Universe is isotropic. This was confirmed, for the mean temperature of the cosmic microwave background $(C M B)$, by the FIRAS experiment on the COBE satellite (Wright et al. 1992; Bennett et al. 1996). However, the higher precision, measurements from the Wilkinson Microwave Anisotropy Probe (WMAP) satellite (Bennett et al. 2003; Hinshaw et al. 2006; Jarosik et al. 2006; Page et al.

\footnotetext{
${ }^{1} \mathrm{KICP}$ and EFI, University of Chicago, Chicago, IL 60637
} 
2006; Spergel et al. 2006), have an anomalously asymmetric distribution, in the temperature fluctuation statistics, between the northern and southern hemispheres of the sky (Eriksen et al. 2004; Hansen et al. 2004a; Vielva et al. 2004; Park 2004; Copi et al. 2004; Hansen et al. 2004b; Larson \& Wandelt 2004; Cruz et al. 2005; Land \& Magueijo 2005; Hansen 2004; Bernui et al. 2005, 2006). On scales greater than about $5^{\circ}$, the variance of the $C M B$ temperature fluctuations is anomalously higher in the southern hemisphere, in both galactic and ecliptic coordinates, compared to the northern hemisphere (Eriksen et al. 2004; Hansen et al. 2004a). This asymmetry also appears in higher order statistics (Vielva et al. 2004; Park 2004; Copi et al. 2004; Hansen et al. 2004b; Larson \& Wandelt 2004; Cruz et al. 2005; Land \& Magueijo 2005; Hansen 2004; Bernui et al. 2005, 2006).

In a spherical harmonic representation, scales ranging from $\ell=2$ to $\ell=40$ were found to be asymmetric. When optimized over direction, only $0.1 \%$ of isotropic simulations were found to produce higher levels of asymmetry (Hansen et al. 2004a).

The result is not sensitive to the frequency band of the $C M B$ (Hansen et al. 2004a) and a similar pattern (at lower significance) is seen in COBE (Hansen et al. 2004a). This argues against a foreground or systematics explanation.

Although a simple single field inflation model would give isotropically distributed perturbations, this is not necessarily the case in multi-field models (Linde \& Mukhanov 2006). Thus, if it can be shown that the $C M B$ fluctuations are not isotropic, it may be an indication that inflation was a multi-field process.

The layout of the paper is as follows: In Sec. 2, a linearly modulated primordial power spectrum is proposed as the source of the observed isotropy breaking. Then, in Sec. 3, a method of evaluating the linear modulation parameters is outlined. The constraints are given in Sec. 4 and their implications and relation to other results are discussed in Sec. 5.

\section{Linear Modulation}

An isotropy-breaking mechanism may be parameterized as (Prunet et al. 2005; Gordon et al. 2005; Spergel et al. 2006)

$$
\delta T(\hat{\mathbf{n}})=\delta T_{\text {iso }}(\hat{\mathbf{n}})(1-f(\hat{\mathbf{n}}))
$$

where $\delta T$ is the observed $C M B$ temperature perturbations, $\delta T_{\text {iso }}$ are the underlying isotrop-

ically distributed temperature perturbations and $\hat{\mathbf{n}}$ is the direction of observation. An isotropic distribution of perturbations is recovered when $f=0$. 
Spergel et al. (2006) parameterized $f$ as

$$
f(\hat{\mathbf{n}})=\sum_{\ell=1}^{j} \sum_{m=-\ell}^{\ell} f_{\ell m} Y_{\ell m}(\hat{\mathbf{n}})
$$

where $Y_{\ell m}$ are spherical harmonics and $j=1$ and $j=2$ were tried. As $f$ will be a real function, the condition $f_{\ell m}=(-1)^{m} f_{\ell-m}^{*}$ is required, where the asterisk indicates the complex conjugate. The effective chi squared improvement, $\Delta \chi_{\text {eff }}^{2} \equiv-2 \Delta \log \mathcal{L}$ is -3 for the $j=1$ case and -8 for the $j=2$ case, where $\mathcal{L}$ is the likelihood (Spergel et al. 2006).

The dipolar modulation $(j=1)$ case has the potential to explain the lack of isotropy between two hemispheres, as it will reduce the variance of the perturbations in one hemisphere and increase it in the other.

In this article, an underlying spatial model for a dipolar modulation is investigated. On large scales $(\ell<30)$ the main contribution ( Hu \& Sugiyama 1995) to the $C M B$ perturbations is the Sachs Wolfe effect (Sachs \& Wolfe 1967)

$$
\frac{\delta T(\hat{\mathbf{n}})}{T} \approx \frac{1}{3} \Phi(\hat{\mathbf{n}})
$$

where $\Phi$ is the curvature perturbation, in the Newtonian gauge (Bardeen 1980), evaluated at last scattering. ${ }^{1}$ The left hand side is the measured temperature fluctuation divided by the average measured temperature.

It follows that on large scales and at last scattering

$$
\Phi=(1-f) \Phi_{\text {iso }} .
$$

A dipolar modulation of the last scattering surface could result from a spatially linear modulation

$$
f(\hat{\mathbf{n}})=\mathbf{w} \cdot \hat{\mathbf{n}}
$$

where $\mathbf{w} \equiv\left(w_{x}, w_{y}, w_{z}\right)$ is the gradient of the modulating function and the dot indicates a dot product.

The spherical harmonic representation is related to a Cartesian representation by

$$
\left[f_{10}, \operatorname{Re}\left(f_{11}\right), \operatorname{Im}\left(f_{11}\right)\right]=\sqrt{\frac{\pi}{3}}\left[2 w_{z},-\sqrt{2} w_{x}, \sqrt{2} w_{y}\right]
$$

As they are linearly related, a uniform prior on one translates into a uniform prior on the other.

\footnotetext{
${ }^{1}$ This is the surface where the Universe becomes effectively transparent to photons and occurs at a redshift of about 1000 .
} 


\section{Likelihood Analysis}

The likelihood is assumed to follow a multivariate Gaussian distribution

$$
\mathcal{L} \propto|\mathbf{C}|^{-1 / 2} \exp \left(-\frac{1}{2} \mathbf{T}^{\mathrm{T}} \mathbf{C}^{-1} \mathbf{T}\right)
$$

where $\mathbf{T}$ is a vector of the temperature measurements in unmasked areas of the $C M B$ pixelized map. Each element of the covariance matrix, $\mathbf{C}$, is evaluated by

$$
C(\hat{\mathbf{n}}, \hat{\mathbf{m}})=\left(1-f(\hat{\mathbf{n}}) C_{\text {iso }}(\hat{\mathbf{n}}, \hat{\mathbf{m}})(1-f(\hat{\mathbf{m}}))+\lambda C_{\operatorname{marg}}(\hat{\mathbf{n}}, \hat{\mathbf{m}})\right.
$$

where $C(\hat{\mathbf{n}}, \hat{\mathbf{m}})$ corresponds to the covariance between pixels at position $\hat{\mathbf{n}}$ and $\hat{\mathbf{m}}$. The instrumental noise is negligible on large scales (Jarosik et al. 2006; Hinshaw et al. 2006) and so is not included.

A marginalization term (Tegmark et al. 1998; Bond et al. 1998; Slosar et al. 2004; Slosar \& Seljak 2004; Hinshaw et al. 2006) for foregrounds and a modulated and unmodulated monopole and dipole was also added

$$
C_{\mathrm{marg}}=\frac{1}{2}\left(C_{0}+C_{1}\right)+\frac{1}{2}(1-f)\left(C_{0}+C_{1}\right)(1-f)+C_{\text {foregrounds }} .
$$

The unmodulated monopole and dipole terms are needed to account for any residual effects of the background temperature and peculiar motion of the observer (Sachs \& Wolfe 1967). When $\lambda$ is made sufficiently large, the likelihood becomes insensitive to any terms included in $C_{\text {marg }}$ (Tegmark et al. 1998; Bond et al. 1998; Slosar et al. 2004; Slosar \& Seljak 2004; Hinshaw et al. 2006).

Third year $W M A P$ data were used $^{2}$ and the preprocessing followed was the same as in the WMAP analysis (Hinshaw et al. 2006; Spergel et al. 2006) for their large scale likelihood evaluation. The $N_{\text {side }}=512$ "Internal Linear Combination" ( $\left.I L C\right)$ map ${ }^{3}$ was masked with the Kp2 mask and smoothed with a $7.3^{\circ}$ (FWHM) Gaussian smoothing function. It was then degraded to $N_{\text {side }}=8$ using the HEALPix ${ }^{4}$ software package (Górski et al. 2005). The Kp2 mask, consisting of zeros and ones, was also degraded to $N_{\text {side }}=8$ and any pixels with values larger than 0.5 were set to one, else they were set to zero. The smoothed degraded $I L C$ map was then remasked with the degraded Kp2 mask. The foreground template was

\footnotetext{
${ }^{2}$ Obtained from http://lambda.gsfc.nasa.gov/product/map/current/

${ }^{3}$ number of pixels $=12 N_{\text {side }}^{2}$.

${ }^{4}$ http://healpix.jpl.nasa.gov
} 
taken to be the difference between the raw $\mathrm{V}$-band map and the $I L C$ map. The foreground marginalization term in Eq. (9) was set equal to the outer product of the foreground template with itself (Tegmark et al. 1998; Bond et al. 1998; Slosar et al. 2004; Slosar \& Seljak 2004; Hinshaw et al. 2006).

The $C_{\ell}$ values were treated as free parameters for $\ell=2$ to 10 . For $\ell=11$ to 32 , the unmodulated maximum likelihood values were used. The smoothing and degrading of the data make the likelihood insensitive to $\ell>32$.

The likelihood, for the modulated model, was numerically maximized using a quasiNewton method.

Marginalized distributions of the parameters were obtained using the Metropolis algorithm. After an initial burn in run, a proposal covariance matrix was constructed from 20000 samples. This was used to generate three additional sets of 20000 samples, each with a different starting value chosen from a burned in chain. The Gelman and Rubin test were used to check convergence and then the 60000 samples were used to evaluate the marginal probability distributions of the parameters. All priors were taken to be uniform. The upper and lower limit of each confidence interval was chosen so as to exclude the same number of samples above and below the interval.

\section{Results}

The improvement, in the likelihood, compared to an unmodulated model was $\Delta \chi_{\text {eff }}^{2}=-9$ with three extra parameters $\left(w_{1}, w_{2}, w_{3}\right)$. A plot of the maximum likelihood modulation function $f$, is shown in Figure 1. The marginalized distributions of $C_{2}$ to $C_{10}$ were found not to be significantly different from those in an unmodulated model (Hinshaw et al. 2006). The weight vector samples $\left(w_{x}, w_{y}, w_{z}\right)$ were converted into Galactic co-latitude (varying between $0^{\circ}, 180^{\circ}$ ), longitude (varying between $-180^{\circ}$ and $180^{\circ}$ ) and percentage change of temperature variance in the direction of symmetry breaking $\left(\Delta \equiv 200\left(w_{x}^{2}+w_{y}^{2}+w_{z}^{2}\right)\right)$. The two dimensional confidence intervals for the co-latitude and longitude are shown in Figure 2. The marginalized one dimensional distributions are shown in Figure 3. Only $0.2 \%$ of the samples were more than $90^{\circ}$ from the maximum likelihood point. The results are summarized in Table 1. 


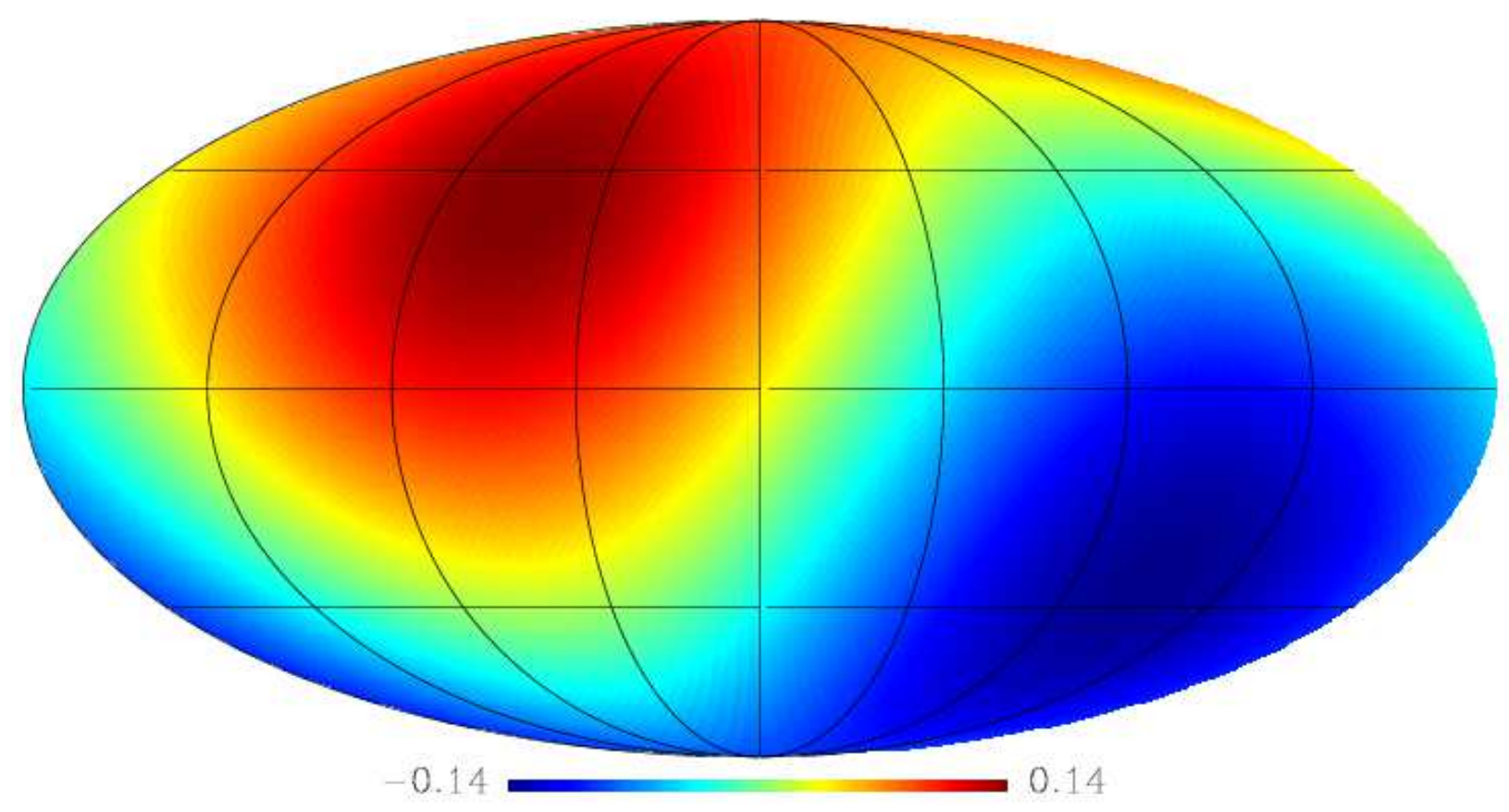

Fig. 1.- Maximum likelihood linear modulation function projected on the last scattering surface. High values indicate suppression of the primordial perturbations and low values indicate enhancement.

Table 1. Marginalized Statistics

\begin{tabular}{ccl}
\hline \hline Parameter & Mean $^{\mathrm{a}}$ & $M L^{\mathrm{b}}$ \\
\hline$\Delta$ & $62_{-18-35-47}^{+18+35+57}$ & 57 \\
Colatitude & $56_{-17-36+65}^{+17+35-51}$ & 51 \\
Longitude & $63_{-26-58-213}^{+28+59+105}$ & 68 \\
\hline
\end{tabular}

${ }^{a}$ Including confidence intervals $(68 \%, 95 \%, 99.7 \%)$.

${ }^{\mathrm{b}}$ Maximum Likelihood. 


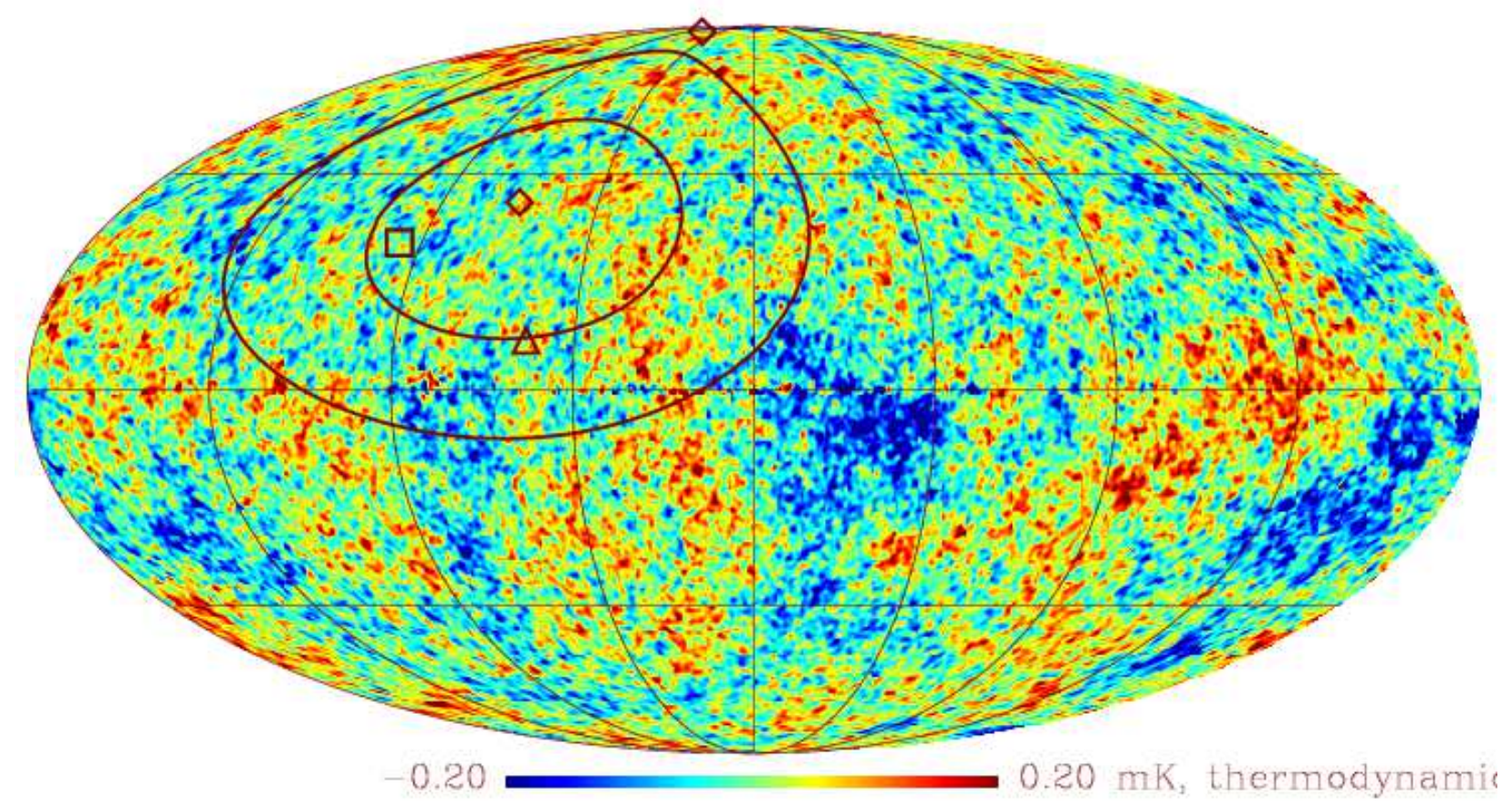

Fig. 2.- The contours enclosing $68 \%$ and $95 \%$ of the Monte Carlo samples. The maximum likelihood $(\diamond)$ Galactic co-latitude and longitude is $\left(51^{\circ}, 68^{\circ}\right)$. One of the most asymmetric directions found by Hansen et al. (2004a) $(\triangle)$ is $\left(80^{\circ}, 57^{\circ}\right)$. The north ecliptic $(\square)$ is at $\left(60^{\circ}, 96^{\circ}\right)$. This is plotted over the third year $N_{\text {side }}=512 I L C$ map (Hinshaw et al. 2006).
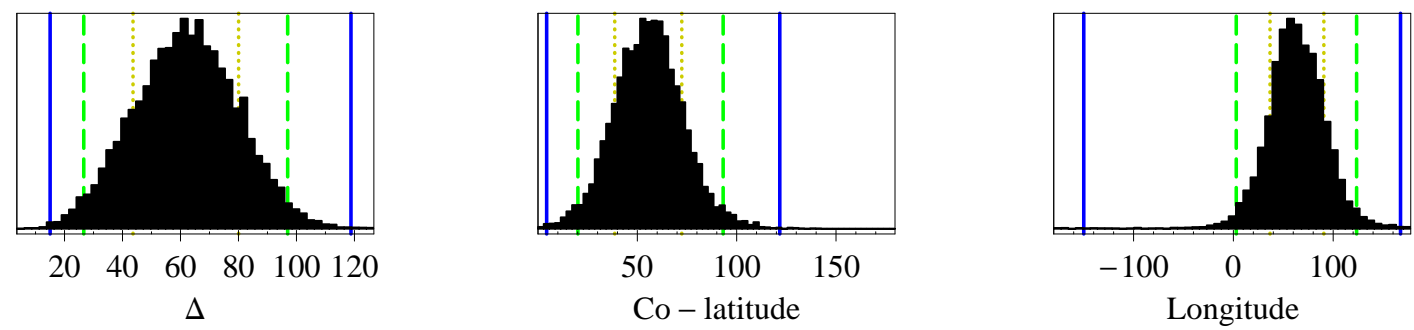

Fig. 3.- Marginalized distributions of the percentage change in the variance across the poles $(\Delta)$ and the galactic coordinates of the direction of the modulation. The $68 \%, 95 \%$ and $99.7 \%$ confidence intervals are also shown. 


\section{Discussion and Conclusions}

Including marginalization over an unmodulated monopole and dipole improved the $\Delta \chi_{\text {eff }}^{2}$ value from -3 to -9 . Marginalization over foregrounds did not play a big role. Similar improvements are obtained when the foreground corrected $\mathrm{V}$ band is used instead of marginalization. Also, the results are not sensitive to the exact method of degrading and applying the mask. A Kp2 extended mask (Eriksen et al. 2006) did not make a significant difference. Including additional $C_{\ell}$, with $\ell>10$, as parameters to be estimated (rather than set to their unmodulated $M L$ values), did not significantly effect the results.

It is interesting to compare the estimated modulation found in this article to that of Hansen et al. (2004a). The 10 most effective axes of symmetry breaking, for a range of scales, are plotted in their Figure 24. A similar area, to the two dimensional confidence intervals in Figure 2, is covered. Also, their Figure 19 compares the power spectra in different north and south hemispheres. The range of values is consistent with the confidence intervals for $\Delta$ in Table 1 and Figure 3.

Prunet et al. (2005) tested for a dipolar modulation. However, the largest scale they looked at was $\ell=20$ to 100 binned. They did not get significant results in that range. As the observed modulation only occurs for $\ell \lesssim 40$ (Hansen et al. 2004a), the $\ell=20$ to 100 range would not be expected to show significant modulation when binned.

Freeman et al. (2006) propose that the modulation of $\ell=2$ to $\ell=7$ may be sensitive to any residual unmodulated dipole component. This is not a concern for the approach taken in this article as an unmodulated dipole is projected out of the likelihood, see Eq. (9).

Searches for lack of isotropy using a method based on a bipolar expansion of the two point correlation function do not detect the north south asymmetry in the $\ell=2$ to $\ell=40$ range (Hajian \& Souradeep 2006). The linear modulation model could be used to understand why the bipolar estimator is insensitive to this type of isotropy breaking.

A small scale cut off in the modulation implies that a linear modulation of the primordial power spectrum would only apply to wave numbers larger than about $4 \times 10^{-3} h \mathrm{Mpc}^{-1}$. It would be interesting to evaluate whether this modulation would be detectable in future large scale galaxy surveys. However, at a redshift of one the change in the variance at opposite poles would only be about four percent, due to the smaller comoving distance.

A number of attempts have been made to explain the asymmetry in terms of local nonlinear inhomogenities (Moffat 2005; Tomita 2005a,b; Inoue \& Silk 2006). It would be interesting to see if the polarization maps of the $C M B$ (Page et al. 2006) could be used to distinguish local effects from a modulation of the primordial perturbations. 
Primordial magnetic fields (Durrer et al. 1998; Chen et al. 2004; Naselsky et al. 2004) and global topology (Kunz et al. 2006) can also lead to isotropy breaking. However, in these cases the modulating function is of higher order than dipolar (Gordon et al. 2005).

As seen in Figure 2, the maximum likelihood direction of modulation was found to be about $44^{\circ}$ from the ecliptic north pole. Only about $9 \%$ of the time would two randomly chosen directions be as close, or closer, together. This may be an indication that the modulation is caused by some systematic effect or foreground. However, as can be seen from Figure 2, the confidence intervals, for the direction of modulation, cover just under half the northern hemisphere. Therefore, the actual direction, of modulation may be significantly further away from the ecliptic north pole.

Standard single field inflation would produce isotropic perturbations. However, multifield models, such as in the curvaton scenario (Lyth \& Wands 2002; Mollerach 1990; Linde \& Mukhanov 1997; Enqvist \& Sloth 2002; Moroi \& Takahashi 2001), can produce, what to a particular observer appear to be, non-isotropic perturbations (Linde \& Mukhanov 2006). The curvaton mechanism produces a web like structure in which relatively stable domains are separated by walls of large nonlinear fluctuations. If the mass of the curvaton field is sufficiently small, our observable Universe could be enclosed within a stable domain. If we happen to live near one of the walls, of a domain, then the amplitude of the perturbations will be larger on the side of the observed Universe closer to the wall (Linde \& Mukhanov 2006). However, if our observed Universe was far enough away from the web walls, the very large scale fluctuations would be linear and so isotropy would be unlikely to appear to be broken (Lyth 2006). As the non-isotropic nature only extends to about $\ell=40$ (Hansen et al. 2004a), it would be necessary for the inflaton perturbations to dominate over the curvaton ones for wave numbers larger than about $4 \times 10^{-3} h \mathrm{Mpc}^{-1}$. The curvaton produces curvature perturbations proportional to $V^{1 / 2}$ (Lyth \& Wands 2002), where $V$ denotes the inflaton potential. While the inflaton produces curvature perturbations proportional to $V^{3 / 2} / V^{\prime}$, where $V^{\prime}$ denotes the slope of the potential. So if there is a sudden drop in $V$ and $V^{\prime}$, it is possible for the non-isotropic curvaton perturbations to dominate for wave numbers smaller than $4 \times 10^{-3} h \mathrm{Mpc}^{-1}$ and inflaton perturbations to dominate for larger wave numbers.

There are oscillations in the $W M A P$ power spectrum, at around $\ell=40$, which may be caused by a change of slope in the inflaton potential (Covi et al. 2006). Whether all these elements can be put together to make a working curvaton model, that fits the data as well as a linear modulation, is still being investigated.

The results presented here give a specific parameterization for the observed asymmetry between the north and south hemispheres of the WMAP data. Having a specific model for the primordial fluctuations will make it easier to develop new tests for this asymmetry and 
help determine if it is a genuine window into new physics at the largest observable scales.

I thank Olivier Doré, Wayne Hu, Dragan Huterer, Nemanja Kaloper, David Lyth and Hiranya Peiris for useful discussions. I was supported by the KICP under NSF PHY0114422. Some of the results in this paper have been derived using the HEALPix (Górski et al. 2005) package. The WMAP data were obtained from the Legacy Archive for Microwave Background Data Analysis ( $L A M B D A)$.

\section{REFERENCES}

Bardeen, J. M. 1980, Phys. Rev. D, 22, 1882

Bennett, C. L., Banday, A. J., Gorski, K. M., Hinshaw, G., Jackson, P., Keegstra, P., Kogut, A., Smoot, G. F., Wilkinson, D. T., \& Wright, E. L. 1996, ApJ, 464, L1+

Bennett, C. L., Halpern, M., Hinshaw, G., Jarosik, N., Kogut, A., Limon, M., Meyer, S. S., Page, L., Spergel, D. N., Tucker, G. S., Wollack, E., Wright, E. L., Barnes, C., Greason, M. R., Hill, R. S., Komatsu, E., Nolta, M. R., Odegard, N., Peiris, H. V., Verde, L., \& Weiland, J. L. 2003, ApJS, 148, 1

Bernui, A., Mota, B., Reboucas, M. J., \& Tavakol, R. 2005, astro-ph/0511666

Bernui, A., Villela, T., Wuensche, C. A., Leonardi, R., \& Ferreira, I. 2006, astro-ph/0601593

Bond, J. R., Jaffe, A. H., \& Knox, L. 1998, Phys. Rev. D, 57, 2117

Chen, G., Mukherjee, P., Kahniashvili, T., Ratra, B., \& Wang, Y. 2004, ApJ, 611, 655

Copi, C. J., Huterer, D., \& Starkman, G. D. 2004, Phys. Rev. D, 70, 043515

Covi, L., Hamann, J., Melchiorri, A., Slosar, A., \& Sorbera, I. 2006, astro-ph/0606452

Cruz, M., Martínez-González, E., Vielva, P., \& Cayón, L. 2005, MNRAS, 356, 29

Durrer, R., Kahniashvili, T., \& Yates, A. 1998, Phys. Rev. D, 58, 123004

Enqvist, K. \& Sloth, M. S. 2002, Nuclear Physics B, 626, 395

Eriksen, H. K., Hansen, F. K., Banday, A. J., Górski, K. M., \& Lilje, P. B. 2004, ApJ, 609, 1198 
Eriksen, H. K., Huey, G., Saha, R., Hansen, F. K., Dick, J., Banday, A. J., Gorski, K. M., Jain, P., Jewell, J. B., Knox, L., Larson, D. L., O’Dwyer, I. J., Souradeep, T., \& Wandelt, B. D. 2006, astro-ph/0606088

Freeman, P. E., Genovese, C. R., Miller, C. J., Nichol, R. C., \& Wasserman, L. 2006, ApJ, 638,1

Gordon, C., Hu, W., Huterer, D., \& Crawford, T. 2005, Phys. Rev. D, 72, 103002

Górski, K. M., Hivon, E., Banday, A. J., Wandelt, B. D., Hansen, F. K., Reinecke, M., \& Bartelmann, M. 2005, ApJ, 622, 759

Hajian, A. \& Souradeep, T. 2006, astro-ph/0607153

Hansen, F. K. 2004, astro-ph/0406393

Hansen, F. K., Banday, A. J., \& Górski, K. M. 2004a, MNRAS, 354, 641

Hansen, F. K., Cabella, P., Marinucci, D., \& Vittorio, N. 2004b, ApJ, 607, L67

Hinshaw, G., Nolta, M. R., Bennett, C. L., Bean, R., Dore', O., Greason, M. R., Halpern, M., Hill, R. S., Jarosik, N., Kogut, A., Komatsu, E., Limon, M., Odegard, N., Meyer, S. S., Page, L., Peiris, H. V., Spergel, D. N., Tucker, G. S., Verde, L., Weiland, J. L., Wollack, E., \& Wright, E. L. 2006, astro-ph/0603451

Hu, W. \& Sugiyama, N. 1995, ApJ, 444, 489

Inoue, K. T. \& Silk, J. 2006, ArXiv Astrophysics e-prints

Jarosik, N., Barnes, C., Greason, M. R., Hill, R. S., Nolta, M. R., Odegard, N., Weiland, J. L., Bean, R., Bennett, C. L., Dore', O., Halpern, M., Hinshaw, G., Kogut, A., Komatsu, E., Limon, M., Meyer, S. S., Page, L., Spergel, D. N., Tucker, G. S., Wollack, E., \& Wright, E. L. 2006, astro-ph/0603452

Kunz, M., Aghanim, N., Cayon, L., Forni, O., Riazuelo, A., \& Uzan, J. P. 2006, Phys. Rev. D, 73,023511

Land, K. \& Magueijo, J. 2005, MNRAS, 357, 994

Larson, D. L. \& Wandelt, B. D. 2004, ApJ, 613, L85

Linde, A. \& Mukhanov, V. 1997, Phys. Rev. D, 56, 535

—. 2006, Journal of Cosmology and Astro-Particle Physics, 4, 9 
Lyth, D. H. 2006, Journal of Cosmology and Astro-Particle Physics, 6, 15

Lyth, D. H. \& Wands, D. 2002, Physics Letters B, 524, 5

Moffat, J. W. 2005, Journal of Cosmology and Astro-Particle Physics, 10, 12

Mollerach, S. 1990, Phys. Rev. D, 42, 313

Moroi, T. \& Takahashi, T. 2001, Physics Letters B, 522, 215

Naselsky, P. D., Chiang, L.-Y., Olesen, P., \& Verkhodanov, O. V. 2004, ApJ, 615, 45

Page, L., Hinshaw, G., Komatsu, E., Nolta, M. R., Spergel, D. N., Bennett, C. L., Barnes, C., Bean, R., Dore', O., Halpern, M., Hill, R. S., Jarosik, N., Kogut, A., Limon, M., Meyer, S. S., Odegard, N., Peiris, H. V., Tucker, G. S., Verde, L., Weiland, J. L., Wollack, E., \& Wright, E. L. 2006, astro-ph/0603450

Park, C.-G. 2004, MNRAS, 349, 313

Prunet, S., Uzan, J.-P., Bernardeau, F., \& Brunier, T. 2005, Phys. Rev. D, 71, 083508

Sachs, R. K. \& Wolfe, A. M. 1967, ApJ, 147, 73

Slosar, A. \& Seljak, U. 2004, Phys. Rev. D, 70, 083002

Slosar, A., Seljak, U., \& Makarov, A. 2004, Phys. Rev. D, 69, 123003

Spergel, D. N., Bean, R., Dore', O., Nolta, M. R., Bennett, C. L., Hinshaw, G., Jarosik, N., Komatsu, E., Page, L., Peiris, H. V., Verde, L., Barnes, C., Halpern, M., Hill, R. S., Kogut, A., Limon, M., Meyer, S. S., Odegard, N., Tucker, G. S., Weiland, J. L., Wollack, E., \& Wright, E. L. 2006, astro-ph/0603449

Tegmark, M., Hamilton, A. J. S., Strauss, M. A., Vogeley, M. S., \& Szalay, A. S. 1998, ApJ, 499,555

Tomita, K. 2005a, Phys. Rev. D, 72, 103506

—. 2005b, Phys. Rev. D, 72, 043526

Vielva, P., Martínez-González, E., Barreiro, R. B., Sanz, J. L., \& Cayón, L. 2004, ApJ, 609, 22

Wright, E. L., Meyer, S. S., Bennett, C. L., Boggess, N. W., Cheng, E. S., Hauser, M. G., Kogut, A., Lineweaver, C., Mather, J. C., Smoot, G. F., Weiss, R., Gulkis, S., Hinshaw, G., Janssen, M., Kelsall, T., Lubin, P. M., Moseley, Jr., S. H., Murdock, T. L., Shafer, R. A., Silverberg, R. F., \& Wilkinson, D. T. 1992, ApJ, 396, L13 
This preprint was prepared with the AAS IATEX macros v5.2. 